\title{
A $12 \mathrm{GHz}$ oscillator based on a GaAs HEMT integrated to a HTS resonator
}

\author{
G. Borghs, J. DeBoeck, I. Francois, D. Chambonnet*, C. Belouet*, Y. Crosnier**, J.C. Carru**, \\ D. Chauvel**, L. Arnaud***, H. Boucher***, J.C. Villegier***, S. Nicoletti****, L. Correra**** \\ and J. Jiménez*****
}

\author{
Interuniversitary Micro-Electronics Center, Leuven, Belgium \\ * Alcatel Alsthom Recherche, Marcoussis, France \\ ** Université de Lille, Lille, France \\ *** LETI-CEA/Technologies Avancées, Grenoble, France \\ **** Instituto CNR Lamel, Bologne, Italy \\ ***** Departmento de Fisica de la materia Condensada, Valladolid, Spain
}

\begin{abstract}
A hybrid oscillator operating at $12 \mathrm{GHz}$ and $77 \mathrm{~K}$ was designed and characterised. The design incorporated on a single substrate a passive superconductive circuit and a III-V active device. YBaCuO films (300nm), grown on $\mathrm{MgO}$, had a surface resistance at $77 \mathrm{~K}$ of 0.3 $\mathrm{m} \Omega$ scaled to $12 \mathrm{GHz}$. The DC and RF characteristics of the pseudomorphic HEMT showed minor differences before and after hybridation. The characterisation of a preliminary prototype oscillator including a superconducting resonator patterned on a $\mathrm{MgO}$ substrate, a gold matching network fabricated on $\mathrm{Al}_{2} \mathrm{O}_{3}$ and a transistor wire bonded gave a phase noise as low as - 75 $\mathrm{dBc} / \mathrm{Hz}$ at $10 \mathrm{KHz}$.
\end{abstract}

\section{INTRODUCTION}

Microwave circuits for radar or communications are likely to be the first area of practical applications of high temperature superconductors (HTS). As a matter of fact HTS thin films such as YBaCuO or $\mathrm{TlBaCaCuO}$ have a microwave surface resistance that is orders of magnitude lower than that of metals at liquid nitrogen temperature [1]. That is why a variety of passive devices like resonators, filters, delay lines or antennae have been sucessfully developed. For example, the HTSSE [2] space experiment is aimed at demonstrating the feasibility of incoporating HTS technology into space environment.

In order to impact subsystem or system level applications, it is necessary to have active devices which produce gain with low noise characteristics. To date, current active HTS devices exhibit too high noise to be used in high performance microwave circuits. On the contrary, III-V solid state devices provide these characteristics and have enhanced performance at cryogenic temperatures. Several authors already reported on hybrid devices assembling HTS passive components and III-V active devices [3],[4],[5]. However the schemes with commercial active devices and conventional integration technique show unsatisfactory parasitic noise.

The purpose of this paper is to report on the development of a hybrid oscillator operating at $12 \mathrm{GHz}$ and combining a pseudomorphic HEMT and a microstrip $\mathrm{YBaCuO}$ ring resonator. Both chips are integrated on a single substrate with the so called Flip-Chip or ELO techniques, described hereafter, which take advantage of the low parasitic inductance and capacitance. In a first part the typical parameters of superconductor deposition and resonator patterning are emphasized. The second part details the oscillator design and the characteristics of a first prototype. The third part describes the hybridation processes and their characteristics. Finally the capabilities of the circuit are discussed. 


\section{SUPERCONDUCTOR}

\subsection{Superconducting film deposition}

High quality epitaxial YBCO films with low surface resistance are required for microwave applications. Many research groups fabricate such YBCO films using MOCVD [6], Inverted Cathode Magnetron Sputtering [7] and Pulsed Laser Deposition (PLD) techniques [8], on $\mathrm{MgO}, \mathrm{LaAlO}_{3}$ and $\mathrm{Al}_{2} \mathrm{O}_{3}$. In this study, films were deposited on (100) $\mathrm{MgO}$ by PLD using a pulsed excimer laser in a procedure which has been described elsewhere [9]. MgO substrates were chosen as a good compromise between substrate brittleness, substrate microwave properties and YBCO film quality. The substrate surfaces were characterised prior to deposition by X-Ray Topography and Atomic Force Microscopy (AFM). Their RMS roughness was in the 0.1-0.3 nm range. The substrates, after rincing in toluene, aceton and iso-propanol, were glued onto the heating block with silver paste. The furnace temperature was monitored by a thermocouple clamped inside the heating block. The substrate temperature measured by a pyrometer in frontal view in the wavelength band 8-14 $\mu \mathrm{m}$, was controlled before and after deposition. It was found to be higher than the temperature indicated by the thermocouple by ca. $10^{\circ} \mathrm{C}$.

Films, $300 \mathrm{~nm}$ thick, with a pure $c_{\perp 0}$ texture $\left(c_{\perp}\right.$ texture without in-plane misoriented grains) were routinely grown in the $750-775^{\circ} \mathrm{C}$ temperature range (see figure 1).

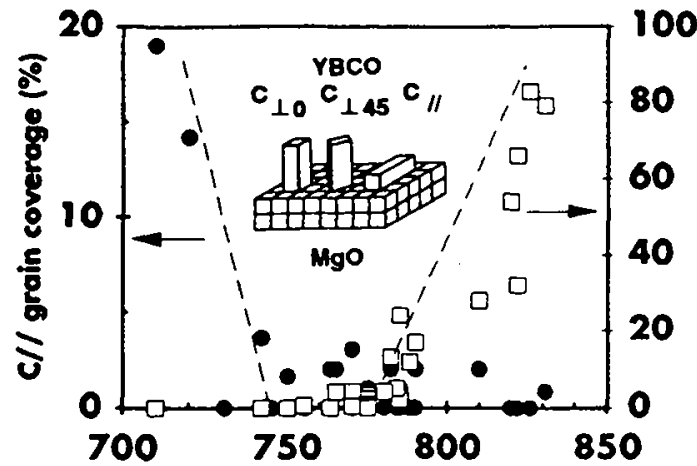

Substrate temperature $\left({ }^{\circ} \mathrm{C}\right)$

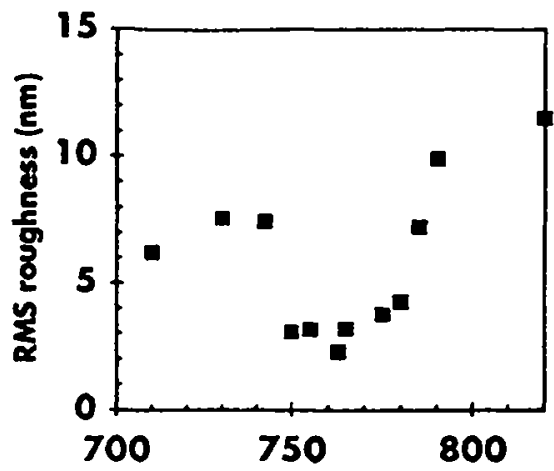

Substrate temperature $\left({ }^{\circ} \mathrm{C}\right)$

Figure 1: Influence of the growth temperature on the crystalline texture (left) and on the RMS roughness (right) of YBCO films $300 \mathrm{~nm}$ in thickness. Broken lines are guides to the eye.

Their RMS roughness, as observed by AFM, was around $2 \mathrm{~nm}$ in this temperature range. After deposition, an annealing procedure was necessary to fully oxidise the films. Typical Tc and Jc( $77 \mathrm{~K})$ values of films thus made were in the range $88-92 \mathrm{~K}$ and $5.10^{5}-2.10^{6} \mathrm{~A} / \mathrm{cm}^{2}$ respectively. The best surface resistance value measured in transmission at $22 \mathrm{GHz}$ and $77 \mathrm{~K}$ on these films was as low as $1 \mathrm{~m} \Omega$ [10].

\subsection{Film patterning and metallisation}

The films were patterned by wet chemical etching using EDTA as the reactant. The procedure uses a standard positive photoresist process. An undercutting of 1 to $2 \mu \mathrm{m}$ was measured and the patterned edge roughness was about $100 \mathrm{~nm}$. Investigation on the patterning induced damages was conducted both by means of micro-Raman spectroscopy with a spatial resolution of less than $1 \mu \mathrm{m}$ and $\mathrm{X}$ Ray Diffraction (XRD) analysis. Negligible damage was observed in XRD spectra. The micro-Raman signal close to the patterned edge was unchanged up to a distance from the edge equal to the spatial resolution. The presence of residual strains, poor oxygen stoichiometry and impurity phases result in significant changes in the Raman spectrum of $\mathrm{YBaCuO}$, mainly frequency shifts and additional peaks [11]. Metallisation steps were necessary for electrical contact and ground plane deposition. Gold contacts were made by sputtering in a lift-off process prior to the etching. $\mathrm{Ti}$ and Au precursor films were sputtered on the back side of the substrates for 
subsequent ground plane deposition. This step was followed by an electrolytic deposition of gold in order to reach a nominal thickness of $4 \mu \mathrm{m}$.

\section{OSCILLATOR}

\subsection{Oscillator design}

The whole oscillator, operating at $12 \mathrm{GHz}$ and $77 \mathrm{~K}$, will be implemented on a single substrate, with metallic ground plane. Passive devices are realized in microstrip technology on a superconducting thin film. The active element is a transplanted pseudomorphic HEMT. The $S$ parameters of this HEMT have been measured at $77 \mathrm{~K}$ to perform the design of the oscillator (figure 2), using simulations on a commercially available software (Microwave Design System).

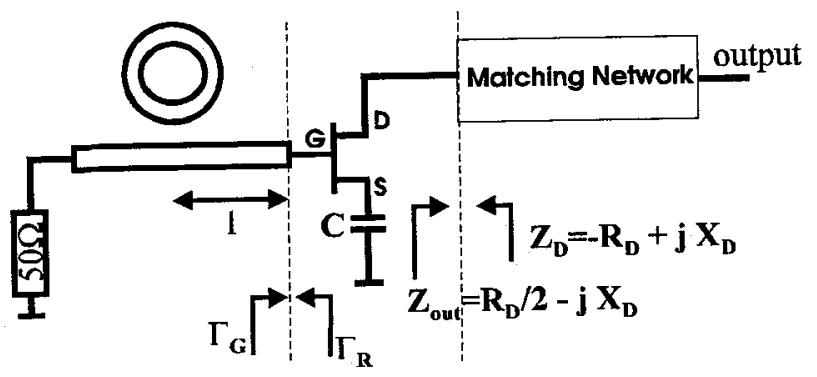

The capacitive feed-back $\mathrm{C}$ at the source terminal is used to obtain a high input reflection coefficient on the gate $\left(\Gamma_{\mathrm{G}}>1\right)$.

The stabilization circuit contains a ring resonator with an adjacent coupling to a $50 \Omega$ line and is loaded with a $50 \Omega$ termination.

Figure 2: Schematic diagram of the superconducting reflection mode oscillator with a transplanted HEMT

Although it probably degrades the loaded quality factor of the resonator, this kind of coupling is favourable to avoid parasitic oscillations and limits additional phase noise. Another reflection oscillator configuration with a ring resonator coupled by a gap at the end of the excitation line is also under study. In the reference plane of the gate of the HEMT, the reflection coefficient of the resonator is expressed as follows :

$$
\Gamma_{R}=\left|\Gamma_{R}\right| \cdot e^{-2 j \beta 1} \quad \text { with } \beta=2 \pi / \lambda_{g}
$$

$\lambda_{\mathrm{g}}=$ guided wavelength and $\mathrm{l}=$ distance between the gate and the ring resonator. If an oscillation occurs, then the following relation will hold at the steady state:

$$
1=\Gamma_{R} \cdot \Gamma_{G}=\left|\Gamma_{R}\right| \cdot e^{-2 j \beta 1} \cdot\left|\Gamma_{G}\right| \cdot e^{j} \arg \left(\Gamma_{G}\right)
$$

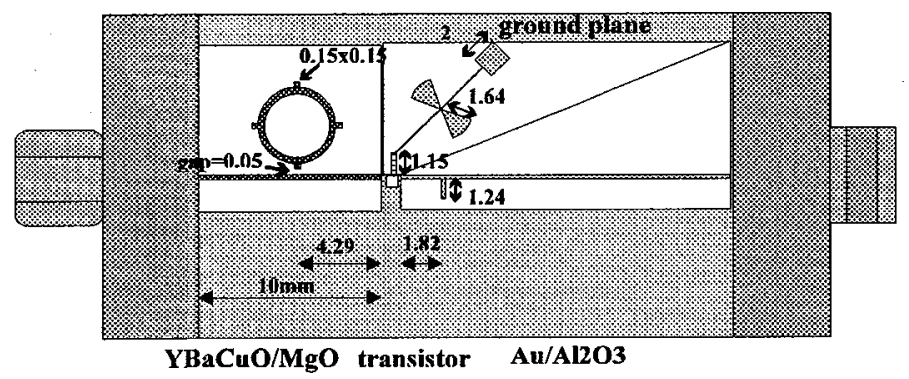

Figure 3: Prototype of the oscillator in its measurement cell.
The length 1 and the resonator coupling gap are also calculated to adjust the phase and the magnitude of $\Gamma_{R}$ for the oscillation condition respectively. The matching network is designed such that the magnitude of the negative real part of the impedance seen by the drain $Z_{D}$ is twice the magnitude of the real part of the impedance of the matching network $\mathrm{Z}_{\text {out }}$.

The prototype already realised, externally biased, differed from the final circuit by the fact that it comprised two different substrates : the superconducting resonant circuit fabricated on a $500 \mu \mathrm{m}$ thick $\mathrm{MgO}$ substrate 
and the gold matching network on a $250 \mu \mathrm{m}$ thick $\mathrm{Al}_{2} \mathrm{O}_{3}$ substrate. An in house transistor was epoxied and connected with bonding wires which were taken into account in the simulations. The layout of this circuit is shown in the figure 3 .

\subsection{Oscillator characterization}

The oscillator shown in the figure 3 , mounted inside a nitrogen cryostat was characterised at $77 \mathrm{~K}$ using a Wiltron Spectrum Analyser. The phase noise, the output power, Pout and the oscillation frequency, fo were directly deduced from the curve of the figure 4 :

fo $=12,3 \mathrm{GHz}$, Pout $=11 \mathrm{dBm}$, Phase noise $=-75 \mathrm{dBc} / \mathrm{Hz}$ at $10 \mathrm{KHz}$ from the carrier. The bias of the transistor was maintained at $\mathrm{Vds}=2 \mathrm{~V}$ and $\mathrm{Ids}=30 \mathrm{~mA}$, leading to an efficiency of $22 \%$..

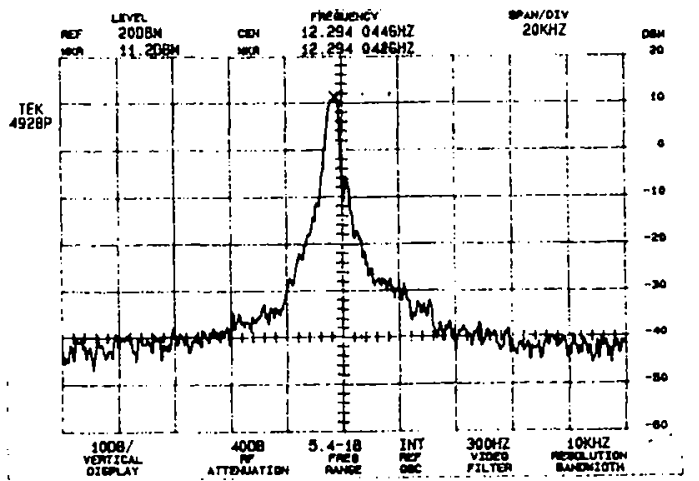

These experimental output power and phase noise are much better than those measured on a similar gold circuit operating at $300 \mathrm{~K}$ and $12 \mathrm{GHz}$. They are comparable to those measured on a fully integrated oscillator operating at $77 \mathrm{~K}$ and $6.5 \mathrm{GHz}$ [4]. The best phase noise already reported $(-125$ $\mathrm{dBc} / \mathrm{hz}$ ) had been measured on a hybrid but not integrated oscillator [5]. The HTS resonator exhibited very high $Q$ factor.

Figure 4: Oscillation spectrum.

\section{HYBRIDATION}

\subsection{Flip-chip bonding technique}

\subsection{1 bonding materials}

In the flip-chip bonding technique [12], a chip is connected onto a substrate via solder bumps as shown in the figure 5 .

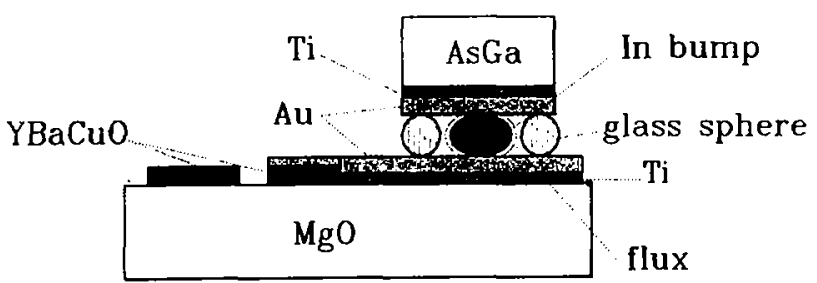

Numerous alloys, having melting temperatures between $100^{\circ} \mathrm{C}$ and $300^{\circ} \mathrm{C}$ have been properly used in the Flip-chip technology [13]. The hybrid oscillator above mentioned, included $\mathrm{YBaCuO}$ patterns and operated in liquid nitrogen.

Figure 5: flip-chip bonding technology.

In order to preserve the oxygen content of the superconducting thin film and thus its properties, bonding process below $150-200^{\circ} \mathrm{C}$ was preferred. In addition good mechanical properties are required for the solder since it is subjected to large stresses during the thermal cycles due to thermal expansion coefficient mismatch between chip and substrate. Both constraints led us to select In solder bumps. This material exhibits the best fatigue life compared to In alloys ${ }^{[14]}$ and a rather low melting point $\left(157^{\circ} \mathrm{C}\right)$. Then, the 
metallic contact pads should have good wettability with $\mathrm{In}$, like $\mathrm{Au}$ or $\mathrm{Cu}$, and low dissolution with solders like, $\mathrm{Pd}, \mathrm{Ni}, \mathrm{Pt}, \mathrm{Ti} . .$. . For all these reasons, a Ti-Au stacking was chosen in the oscillator fabrication.

\subsection{2. bonding process}

In the hybrid oscillator design, the connection bumps were located at the extremities of $50 \Omega$ microstrip lines. The typical widths of these lines were 200-250 $\mu \mathrm{m}$ depending upon the substrate microwave characteristics. In order to match the characteristic impedance as close as possible, In slices $300 \mu \mathrm{m}$ in diameter and $200 \mu \mathrm{m}$ in thickness were fabricated. They were then deposited on the top of the Au patterns. Dielectric spacers were added to maintain the shape of the solder bumps. They consisted of glass spheres glued with an U.V polymerisation glue.

The bonding set up comprised a thermoelectric cooler with inverse biasing able to provide a quick heating and holding the substrate. The upper chip was maintained on a vacuum chuck forced down along the zaxis to perform final alignment through a binocular. In addition a small pressure could be applied to the two aligned samples before or during heating .

\subsection{3 sample performance}

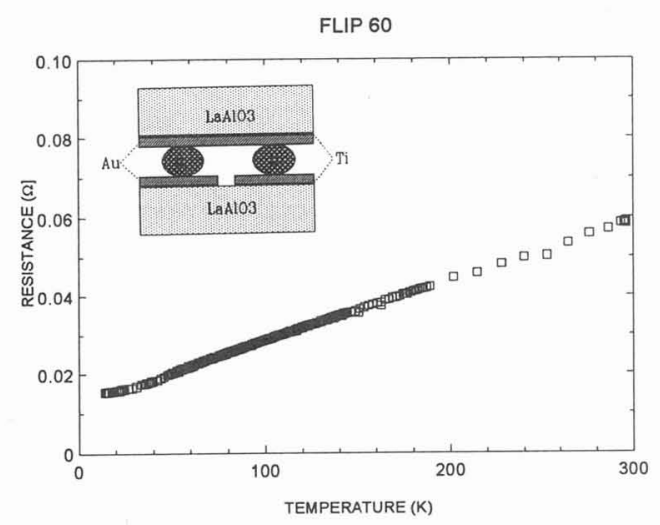

The mechanical properties of the flip-chip structures were investigated during temperature cycles.

The resistance variation of test samples, shown in the figure 6 , were monitored between $77 \mathrm{~K}$ and $300 \mathrm{~K}$. The linear behavior of the resistance value versus temperature meant that the structure remain unchanged during thermal cycles. If not, the resistance would suddenly increased.

The characteristic impedance of a microstrip line having two parts connected in a flip-chip manner as described before is investigated with Time Domain Response. The $50 \Omega$ characteristic impedance of the microstrip gold line patterned on $\mathrm{MgO}$ substrate was locally decreased to $48 \Omega$.

Figure 6: resistance variation of a flip-chip test sample

\subsection{Epitaxial lift-off technique}

The selective etching (with respect to GaAs) of a high quality thin AlAs layer in HF solution has triggered the development of the Epitaxial lift-off technique (ELO) [15]. A high quality epitaxial layer is grown lattice matched with incorporation of an AlAs release layer. After selective etching of the AlAs layer, the wax-covered epitaxial structure is removed from its original substrate and transfered to its new host substrate

Figure 7: Measured $\mathrm{H} 21$ characteristics before (+ to $25 \mathrm{GHz}$ ) and after (- to 67 $\mathrm{GHz}$ ) Epitaxial Lift-Off.

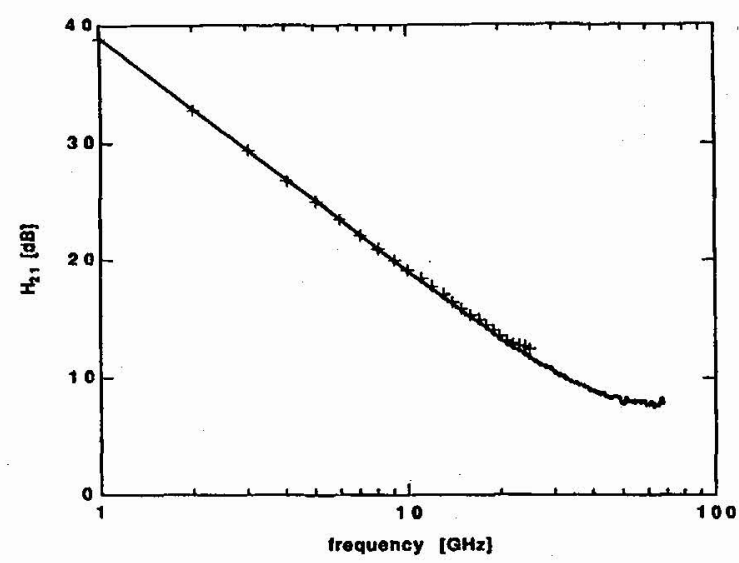


The ELO technique has clearly demonstrated its power in the fabrication of a wide variety of high quality devices on dissimilar substrates and has now entered the stage of technological development [16].GaAs pseudomorphic HEMT with a gate length of $0.25 \mathrm{~mm}$ were lift-off from the GaAs substrate and attached to $\mathrm{MgO}$ or LAaLO3 substrates. Only minor differences were observed in the DC and RF characteristics before and after ELO (see figure 7).

A high extrinsic transition frequency $(95 \mathrm{GHz}$ ) and a high microwave gain (MAG $=16 \mathrm{~dB}$ at $12 \mathrm{GHz}$ ) was measured. Typical phase noise characteristics were measured (NFmin $=1 \mathrm{~dB}$ at $12 \mathrm{GHz}$ ) indicating that the high frequency noise was not affected by the ELO.

\section{CONCLUSION}

A hybrid oscillator integrating a pseudomorphic HEMT and a $\mathrm{YBaCuO}$ ring resonator on a single substrate was designed using the $S$ parameters of the transistor measured at $77 \mathrm{~K}$. The computed frequency of the device was $12 \mathrm{GHz}$ while an experimental frequency of $12.3 \mathrm{GHz}$ was measured. The as grown superconductor film had a surface resistance of $0.3 \mathrm{~m} \Omega$ scaled to $12 \mathrm{GHz}$ at $77 \mathrm{~K}$. The patterning operation was well characterised so that no degradation of the superconductor was observed. The DC and RF characterisation of the hybridized transistor showed minor differences before and after hybridation. The characterisation of a preliminary prototype oscillator including a superconducting resonator patterned on a $\mathrm{MgO}$ substrate, a gold matching network fabricated on $\mathrm{LaAlO}_{3}$ and a transistor wire bonded gave a phase noise as low as $-75 \mathrm{dBc} / \mathrm{Hz}$ at $10 \mathrm{KHz}$. This value allows one to expect even improved performance for the hybrid oscillator integrated on a single substrate.

The authors wish to tanks D. Keller at the Laboratoire de Minéralogie-Cristallographie of the Université Paris VI et VII for AFM measurements.

This work is funded by the ESPRIT Basic Research Action No 6625.

\section{References:}

[1] H. Piel and G. Muller, IEEE Trans magn, MAG 27 (1991), 854.

[2] J. C. Ritter, M. Nisenoff, G. Price and S.A. Wolf, IEEE Trans magn ,MAG 27 (1991), 2553.

[3] R. Kleiber R. Ramish, A. Valenzuela, R. Weigel, P. Russer, IEEE Microwave and Guided Wave Letters, vol. 2 (1992) 22-24.

[4] N. J. Roher, G.J. Valco, K.B. Bhasin; IEEE Trans Microwave Theory Tech., MTT41 (1993), 1865.

[5] Z. Y. Shen, P. Pang, C. Wilker, D. B. Laubacher, W. L. Holstein, C. F. Carter, IEEE Transaction on Applied Superconductivity, vol 3 (1993) 2832-2835.

[6] J.Luine, K.Daly, R.Hu, A.Kain, A.Lee, H.Manasevit, C.Pettiette-Hall, R.Simon. IEEE Trans.Magn., MAG 27, (1991), 1528 .

[7] L. Antognazza, K. Char, T.H. Geballe, L.L.H. King. Appl.Phys. Lett. 63 (7) (1993), 1005.

[8] J.C. Villegier, H. Moriceau, H. Boucher, R. Chicault, L. Di Cioccio, A. Jager, M. Swerdtfeger, M. Vabre, C. Villard, IEEE Trans magn, MAG 27, (1991), 1552.

[9] D. Chambonnet, C. Belouet, C. Fages, M. Puech. in Laser ablation of electronic materials. editors : E.Fogarassy \& S.Lazare (North-Holland, Amsterdam, 1992), p.317.

[10] F. Mehri, P. Lepercq, J.C. Carru, E. Playez, C.Thivet, A. Perrin, D. Chambonnet. Third SEE

Worshop "Supraconducteurs à haute température critique", Caen, 16-17 november 1993.

[11] S. F. Karmenko et al. Supercond. Sci. Technol. 6, (1993), 23.

[12] M. Wale, M. Goodwin, Circuits and Devices (1992) pp 25-31.

[13] K. Pickering, P. Southworth, C. Wort, A. Parsons, and D. J. Pedder, J. Vac. Sci. Tecnol. A 8, (1990), 1503.

[14] K Hashimoto, T Nakanishi, M. Ochiai, Fujitsu Sci. Tech. J. 28 (1992), pp 301-309.

[15] E. Yablonovitch, T. Gmitter, J. P. Harbison, R. Bhat, Appl. Phys. Lett. 51 (1987) 2222.

[16] G. Borghs, J. De Boeck, I. Pollentier, P. Demeester, C. Brys, W. Dobbelare, Proc. 20th

Int. Symp. on GaAs and related compounds, Freibourg 1993. 